\title{
Electrical brain imaging evidences left auditory cortex involvement in speech and non-speech discrimination based on temporal features
}

\author{
Tino Zaehle*1, Lutz Jancke ${ }^{1}$ and Martin Meyer ${ }^{1,2}$
}

Address: ${ }^{1}$ Department of Neuropsychology, University of Zurich, 8050 Zurich, Switzerland and ${ }^{2}$ Institute of Neuroradiology, University Hospital of Zurich, 8091 Zurich, Switzerland

Email: Tino Zaehle* - tino.zaehle@psychologie.unizh.ch; Lutz Jancke - l.jancke@psychologie.unizh.ch;

Martin Meyer - mmeyer@access.unizh.ch

* Corresponding author

Published: 10 December 2007

Behavioral and Brain Functions 2007, 3:63 doi:10.1186/1744-908I-3-63
Received: 4 September 2007

Accepted: 10 December 2007

This article is available from: http://www.behavioralandbrainfunctions.com/content/3/I/63

(C) 2007 Zaehle et al; licensee BioMed Central Ltd.

This is an Open Access article distributed under the terms of the Creative Commons Attribution License (http://creativecommons.org/licenses/by/2.0), which permits unrestricted use, distribution, and reproduction in any medium, provided the original work is properly cited.

\begin{abstract}
Background: Speech perception is based on a variety of spectral and temporal acoustic features available in the acoustic signal. Voice-onset time (VOT) is considered an important cue that is cardinal for phonetic perception.
\end{abstract}

Methods: In the present study, we recorded and compared scalp auditory evoked potentials (AEP) in response to consonant-vowel-syllables (CV) with varying voice-onset-times (VOT) and nonspeech analogues with varying noise-onset-time (NOT). In particular, we aimed to investigate the spatio-temporal pattern of acoustic feature processing underlying elemental speech perception and relate this temporal processing mechanism to specific activations of the auditory cortex.

Results: Results show that the characteristic AEP waveform in response to consonant-vowelsyllables is on a par with those of non-speech sounds with analogue temporal characteristics. The amplitude of the $\mathrm{NIa}$ and $\mathrm{NI}$ b component of the auditory evoked potentials significantly correlated with the duration of the VOT in CV and likewise, with the duration of the NOT in non-speech sounds.

Furthermore, current density maps indicate overlapping supratemporal networks involved in the perception of both speech and non-speech sounds with a bilateral activation pattern during the $\mathrm{NIa}$ time window and leftward asymmetry during the NIb time window. Elaborate regional statistical analysis of the activation over the middle and posterior portion of the supratemporal plane (STP) revealed strong left lateralized responses over the middle STP for both the NIa and NIb component, and a functional leftward asymmetry over the posterior STP for the NIb component.

Conclusion: The present data demonstrate overlapping spatio-temporal brain responses during the perception of temporal acoustic cues in both speech and non-speech sounds. Source estimation evidences a preponderant role of the left middle and posterior auditory cortex in speech and nonspeech discrimination based on temporal features. Therefore, in congruency with recent fMRI studies, we suggest that similar mechanisms underlie the perception of linguistically different but acoustically equivalent auditory events on the level of basic auditory analysis. 


\section{Background}

Auditory language perception is based on a variety of spectral and temporal acoustic information available in the speech signal [1]. One important temporal cue used to distinguish between stop-consonants is the voice onset time (VOT). The VOT, defined as the duration of the delay between release of closure and start of voicing, characterizes voicing differences among stop consonants in a wide variety of languages [2] and can thus be considered one of the most important acoustic cues encoding linguistically relevant information. The perceptual ability of resolving two signals as temporally discrete requires that the brain has a temporally segregated representation of those events.

Electrophysiological studies have consistently demonstrated VOT-related auditory evoked potential (AEP) differences in the N1 component with a single peak in response to short VOTs, and with a double-peaked in response to longer VOTs in humans [3-7], monkey [8,9] and guinea pig [10]. In humans it has been shown that non-speech sounds with related temporal characteristics as consonant-vowel-syllables $(\mathrm{CV})$ resemble these pattern of acoustic temporal processing [11]. In particular, this study showed using intracerebral depth electrodes that the evoked responses of the left, but not the right primary auditory cortex are differential for the processing of voiced and voiceless consonants and their non-speech analogues.

Further support for a general mechanism for encoding and analysing successive temporal changes in acoustic signals has been evidenced by studies demonstrating that patients with acquired brain lesions and aphasia [12,13], children with general language-learning disabilities $[14,15]$ and children and adults with dyslexia [16] show impaired auditory processing of temporal information in non-verbal stimuli. Furthermore, children with reading disabilities are deficient in phoneme perception, which is reflected by inconsistent labelling of tokens in VOT series $[17,18]$, and these children also perform less consistently in labelling of tone onset time tokens [19] and exhibit poorer auditory order thresholds [20]. Moreover, it is known that the ability for phoneme discrimination in these children can be increased by a behavioural training using more salient versions of the rapidly changing elements in the acoustic waveform of speech [21,22].

Recent electrophysiological and neuroimaging studies point to the important role of the primary and secondary auditory cortex for the processing of acoustic features in speech and non-speech sounds. Several investigations using intracranial recording [9,11], scalp EEG [23,24], MEG [25] as well as fMRI [26,27] demonstrated an elevated role of the human primary auditory cortex for the temporal processing of short acoustic cues in speech and non-speech sounds. Furthermore, auditory association areas along the posterior supratemporal plane, in particular the bilateral planum temporale (PT) have also been associated with the processing of rapidly changing auditory information during sub-lexical processing $[26,28,29]$. However, due to BOLD-related limitations in temporal resolutions, the EEG method is far more suitable for elucidating the temporal organization of speech perception. In combination with a recently developed source estimation algorithm [30], it even allows the mapping the spatiotemporal dynamics of elemental aspects of speech perception, i.e. VOT decoding. Thus, the most important goal of this study is the validation of the aforementioned left middle and posterior auditory cortex recruitment in speech and non-speech discrimination based on temporal features.

In the present study, we recorded and compared scalp AEPs in response to CV-syllables and non-speech analogues with varying VOT and noise-onset-time (NOT), respectively. Here we aimed to investigate the neural coding of acoustic characteristics underlying speech perception and relate this temporal processing mechanism to specific activations of the auditory cortex. It has been demonstrated that these processing mechanisms are reflected by modulations of the AEP. The $\mathrm{N} 1$ deflection in particular is an obligatory component considered to reflect the basic encoding of acoustic information of the auditory cortex $[31,32]$. Furthermore, this component reflects the central auditory representation of speech sounds [33,34] and non-speech sounds [35]. Thus, in the context of the present study we focused on the modulations during the N1 time window elicited by brief auditory stimuli that varied systematically along an acoustic and a linguistic dimension. In addition, we examined the extent to which the pattern of neural activation differs in distinct portions of the auditory cortex. As mentioned above, both the middle compartment of the supratemporal plane (STP) accommodating the primary auditory cortex and the posterior compartment of the supratemporal plane harbouring the planum temporale are crucial for processing transient acoustic features in speech and nonspeech sounds. In order to systematically investigate the contribution of these auditory cortex sections, we applied a low-resolution brain electromagnetic tomography (LORETA) approach and predicted functional leftward asymmetric responses to rapidly changing acoustic cues over the middle and posterior portion of the STP.

\section{Methods}

In a behavioural pilot study, 24 healthy, right-handed native speakers of German (mean age $=26.7 \pm 4.56$ years, 13 female) performed a phonetic categorization task. A synthetic VOT continuum was used ranging from 20 to 40 
ms VOT in $1 \mathrm{~ms}$ steps. Participants were instructed to listen to each syllable and to decide whether the syllable was [da] or [ta] by pressing a corresponding button as quickly and accurately as possible. Figure 1 illustrates results of this pilot study. The graph shows the averaged identification curve indicating the percentage of syllables that were identified as /ta/. As illustrated in Figure 1, the mean categorization boundary as indicated by the inflection point of the fitted polynomial function was at a VOT of $30 \mathrm{~ms}$. The results of this behavioural study formed the basis for the subsequent electrophysiological investigation. As a consequence, we used syllables with a VOT of $5 \mathrm{~ms}$, as they were consistently identified as the syllable /da/, a VOT of $60 \mathrm{~ms}$, consistently identified as the syllable /ta/ and syllables with the VOT of $30 \mathrm{~ms}$ reflecting the averaged categorization boundary between / da/ and /ta/. We used a VOT of $5 \mathrm{~ms}$ for the voiced CV-/da/ and a VOT of $40 \mathrm{~ms}$ for the unvoiced CV-/ta/ to ensure the use of VOT stimuli that are clearly in the voiced segment $(5 \mathrm{~ms})$ and in the unvoiced segment (60 ms).

The electrophysiological experiment was conducted in a dimly lit, sound attenuated chamber. Subjects were placed in a comfortable chair at $110 \mathrm{~cm}$ distance from the monitor and scalp recorded event-related potentials (ERPs) in response to $\mathrm{CV}$-syllables and non-speech sounds were obtained from 18 male right-handed, native German speaking healthy volunteers (mean age $=28.6 \pm 3.45$ years). None had any history of hearing, neurological, or psychiatric disorders. After a full explanation of the nature and risks of the study, subjects gave their informed con-

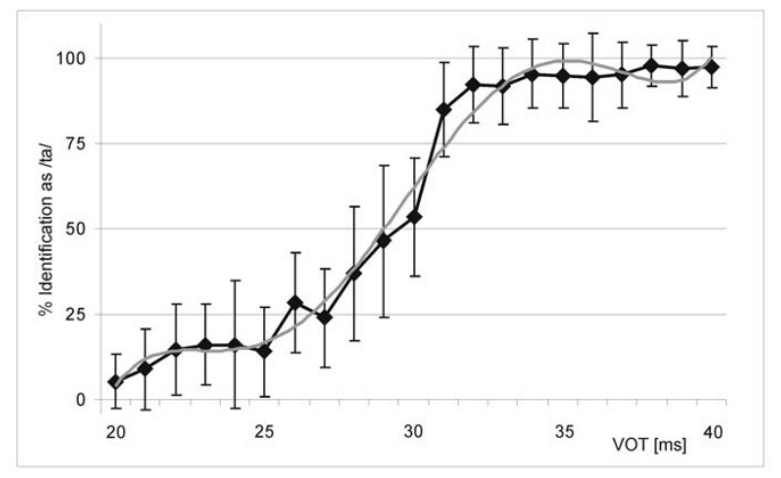

Figure I

Averaged identification curve (+/-I standard deviation) indicating the percentage of CV-syllables that were identified as / $\mathrm{ta} /$ in relation to their VOT (black, diamonds) and fitted polynomial function (gray) $\left[y=0.0011 x^{5}-0.059 x^{4}+1.0989 x^{3}-\right.$ $8.0781 x^{2}+25.458 x$ - 14.507]; Inflection point: $x \mid y$ [10.98|63.86]; corresponding to a VOT of $29.98 \mathrm{~ms}$. sent for the participation according to a protocol approved by the local ethics committee.

The auditory stimuli were generated with a sampling depth of 16 bits and a sampling rate of $44.1 \mathrm{kHz}$ using the SoundForge 4.5 Software [36] and PRAAT [37]. We used a modified version of the stimulus material described by Zaehle et al., (2004) [26]. Figure 2 shows wave-forms of the applied stimuli. Stimuli material consisted of CV syllables with varying voice-onset-times ( $5 \mathrm{~ms}, 30 \mathrm{~ms}$ and 60 $\mathrm{ms})$ as revealed in the pilot behavioural study and analogously, non-speech sounds with varying noise-onsettimes ( $5 \mathrm{~ms}, 30 \mathrm{~ms}$ and $60 \mathrm{~ms}$ ). For the non-speech condition, we created stimuli containing two sound elements separated by a gap. The leading element was a wideband noise burst with a length of $7 \mathrm{~ms}$. The trailing element was a bandpassed noise centred on $1.0 \mathrm{kHz}$ and a width of 500 $\mathrm{Hz}$. The duration of the gap was varied. The duration of each single stimulus was consistent (330 ms). Auditory stimuli were presented binaurally using hi-fi headphones ( $55 \mathrm{~dB}$ sound pressure level). Stimulation and recording of the responses were controlled by the Presentation software (Neurobehavioral Systems, USA).

The EEG experiment comprised ten blocks. Within each block, 18 trials of each stimulus category were presented in a randomized order resulting in presentations of 180 stimuli-pairs. For each trial, volunteers performed a samedifferent discrimination task on a pair of stimuli belonging to one stimulus category. The stimuli varied with respect to the temporal manipulation of the NOT and VOT. Stimuli of one pair were presented with an inter stimulus interval of 1300 ms. Participants indicated their answers by pressing one of two response buttons. We utilized this task to ensure subjects' vigilance throughout the experiment and to engage the subjects to attend to the auditory stimulation. However, we were primarily interested in the electrophysiological responses to acoustic features underlying pure and elemental speech perception. We also aimed to avoid confounds with the neural correlates of decision making instantly following the second stimulus of each pair of VOT and NOT. Thus, only the first stimulus of each stimulus pair was analysed and included into the following analysis.

EEG was recorded from 32 scalp electrodes (30 channels +2 eye channels) located at standard left and right hemisphere positions over frontal, central, parietal, occipital, and temporal areas (subset of international 10/10 system sites: $\mathrm{Fz}, \mathrm{FCz}, \mathrm{Cz}, \mathrm{CPz}, \mathrm{Pz}, \mathrm{Oz}, \mathrm{Fp} 1, \mathrm{Fp} 2, \mathrm{~F} 3, \mathrm{~F} 4, \mathrm{C} 3, \mathrm{C} 4$, P3, P4, O1, O2, F7, F8, T7, T8, P7, P8, TP7, TP8, FT7, FT8, FC3, FC4, CP3, and CP4) using a bandpass of $0.53-70 \mathrm{~Hz}$ with a sampling rate of $500 \mathrm{~Hz}$. We applied sintered silver/silver chloride electrodes (Ag/AgCl) and used the $\mathrm{FCz}$ position as the reference. Impedances of these electrodes 


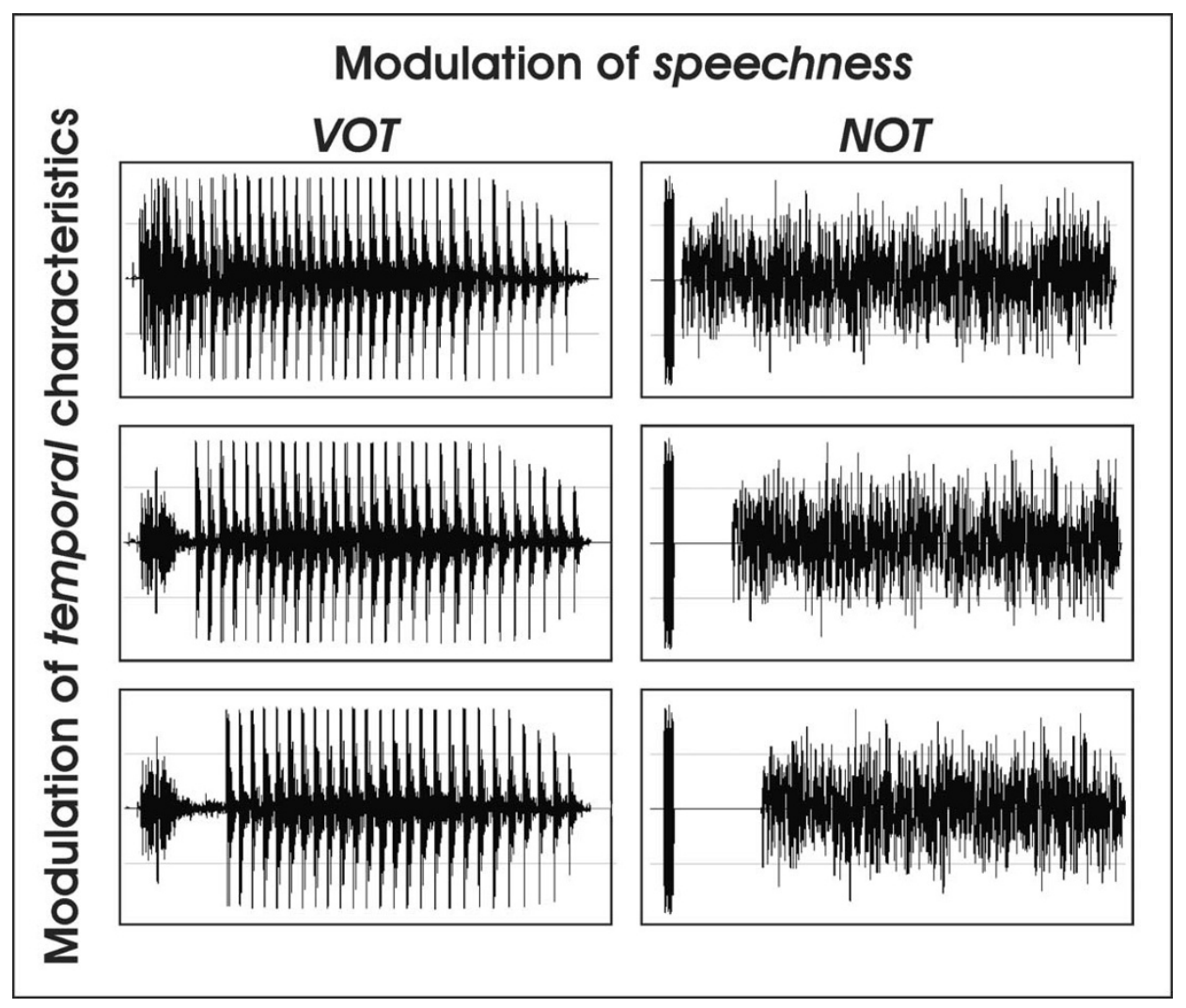

Figure 2

Waveforms of the auditory stimulation. The left panel shows speech stimuli (CV) with varying VOT (5, 30, 60 ms), and the right panel shows non-speech stimuli with varying NOT (top to bottom: 5, 30, $60 \mathrm{~ms}$ ).

were kept below $5 \mathrm{k} \Omega$. Trials containing ocular artefacts, movement artefacts, or amplifier saturation were excluded from the averaged ERP waveforms. The processed data were re-referenced to a virtual reference derived from the average of all electrodes. Each ERP waveform was an average of more than 100 repetitions of the potentials evoked by the same stimulus type. The EEG recordings were sectioned into $600 \mathrm{~ms}$ epochs (100 ms pre-stimulus and 500 ms post-stimulus) and a baseline correction using the prestimulus portion of the signal was carried out. ERPs for each stimulus were averaged for each subject and grandaveraged across subjects.

In order to statistically confirm the predicted differences between AEP components at $\mathrm{Cz}$ as a function of experimental stimuli, mean amplitude ERPs time-locked to the auditory stimulation were measured in two latency windows (110-129 ms and 190-209 ms) determined by visual inspection covering the prominent N1a and N1b components. Analyses of variance (ANOVAs) with factors temporal modulation $(5,30,60 \mathrm{~ms})$ and speechness (VOT/ NOT) were computed for central electrode $(\mathrm{Cz})$, and the $\mathrm{p}$ values reported were adjusted with the GreenhouseGeisser epsilon correction for nonsphericity.

Subsequently, we applied an inverse linear solution approach - LORETA (low-resolution electromagnetic tomography) to estimate the neural sources of eventrelated scalp potentials $[38,39]$. In order to verify the estimated localization of the N1a and N1b component, we calculated the LORETA current density value $\left(\mu \mathrm{A} / \mathrm{mm}^{2}\right)$ for the AEPs within the $3 \mathrm{D}$ voxel space. We used a transformation matrix with high regularization (1e3* (first eigenvalue)) to increase signal to noise ratio. The maxima of the current density distributions were displayed on a cortical surface model and transformed in stereotactic Talairach space [40]. Subsequently, to specifically test the neurofunctional hypothesis of the bilateral middle and posterior STP, we calculated a post hoc region-of-interest (ROI) analysis. We defined four 3D ROIs in STP (left middle STP, right middle STP, left posterior STP, right posterior STP). The landmarks of ROIs were determined by an automatic anatomical labelling procedure implemented in LORETA. We collected mean current density values from each individual and each distinct 3D ROI by means 
of the ROI extractor software tool [41]. The mean current density values for each ROI were submitted to a $3 \times 2 \times 2$ ANOVA with the factors temporal modulation $(5,30,60$ $\mathrm{ms}$ ), hemisphere (left/right) and speechness (VOT/NOT)

\section{Results}

Grand averaged waveforms evoked by each of the three speech and three non-speech stimuli recorded from $\mathrm{Cz}$ are shown in Figure 3. We observed that all stimuli elicited a prominent N1a component with the shortest VOT/NOT modulation $(5 \mathrm{~ms})$ yielding the most enhanced amplitude. Furthermore, we noticed a second negative deflection peaking around $200 \mathrm{~ms}$ after stimulus onset (N1b) also revealing sensitivity to the temporal modulation of the sounds. In order to statistically examine the ERP effects, mean amplitude of the ERP waveforms were measured in two 20 ms latency windows.

Results of the $3 \times 2$ ANOVA with the factors temporal modulation $(5,30,60 \mathrm{~ms})$ and speechness (VOT/NOT) for the N1a (TW I: 110-129 ms latency window) revealed a significant main effect of the factor temporal modulation $(\mathrm{F}(1.77,30.1)=12.45, \mathrm{p}<0.001)$. Similarly, the N1b (190-209 ms latency window) ANOVA revealed a signifi-

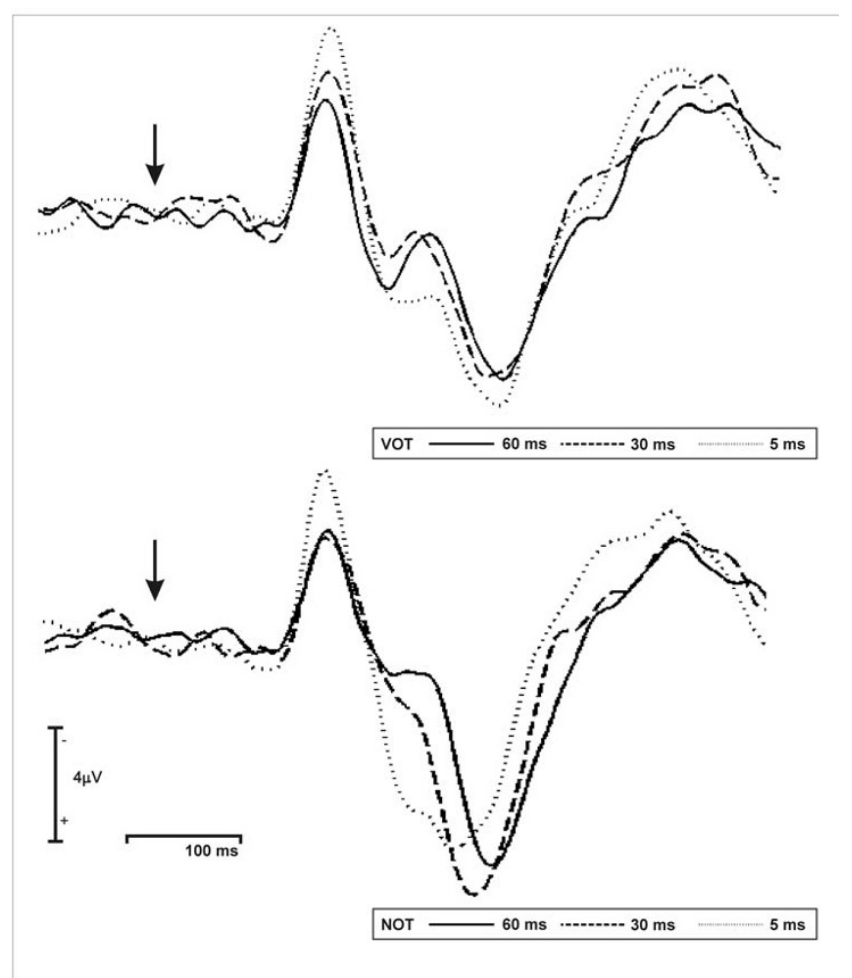

\section{Figure 3}

Averaged electrophysiological data, recorded from 18 participants time locked at the onset of stimulation at central $(\mathrm{Cz})$ electrode during the perception of VOT (top) and NOT stimuli. cant main effect of the factor temporal modulation ( $\mathrm{F}(1.58$, $26.92)=15.7, p<0.001)$. Furthermore, the ANOVA for the N1b also revealed a significant main effect of the factor speechness $(\mathrm{F}(1,17)=19.88, \mathrm{p}<0.001)$ and a significant temporal modulation by speechness interaction ( $\mathrm{F}(1.6$, $27.4)=4.79, \mathrm{p}<0.05)$.

Subsequently, post-hoc analyses were conducted separately for the speech and non-speech stimulation. Figure 4 shows plots of mean amplitude of the temporal modulation separated for speech and non-speech for a) N1a and b) N1b. The results of the one-factorial ANOVAs are listed in Table 1. For the N1 (110-129 ms latency), separate one-factorial ANOVA revealed a significant main effect of the factor temporal modulation for the non-speech sounds $(\mathrm{F}(1.8,30.9)=8.14 \mathrm{p}<0.001)$. Test for linear contrast demonstrated a significant linear relationship of the N1a mean amplitude and length of the NOT in the non-speech sounds $(F(1,17)=15.53, p=0.001)$. Similarly, one - factorial ANOVAs with the factor temporal modulation in the speech sounds revealed a significant main effect $(\mathrm{F}(1.61$, $27.4)=5.34, \mathrm{p}<0.05$ ) and test for linear contrast revealed significant linear relationship of the N1a mean amplitude and length of the VOT in the speech sounds $(F(1,17)=$ $9.39, \mathrm{p}<0.05)$. The same pattern of activation was present at the $190-209$ ms latency window (N1b). Separate onefactorial ANOVAs revealed a significant main effect of the factor temporal modulation for the non-speech sounds $(\mathrm{F}(1.23,21.1)=18.09, \mathrm{p}<0.001)$, and a one-factorial ANOVA with the factor temporal modulation revealed a significant main effect $(F(1.79,30.49)=3.85, \mathrm{p}<0.05)$ for the speech sounds. Tests for linear contrast revealed a significant linear relationship of the N1b mean amplitude and length of the NOT in the non-speech sounds $(\mathrm{F}(1,17)$ $=24.18, \mathrm{p}<0.001)$, and $V O T$ in the speech sounds $(\mathrm{F}(1,17)=4.99, \mathrm{p}<0.05)$.

Results for the source localization analysis are presented in Table 2. The table lists coordinates and corresponding brain regions associated with current density maxima for the speech and non-speech sounds obtained separately for the N1a and N1b time windows. As shown in Figure 5, for the N1a time window current density maps indicate that left and right posterior perisylvian areas contribute to both speech and non-speech sounds. With regard to the N1b, source estimation showed enlarged current density distribution over the left posterior STP and the anterior cingulate gyrus for speech and non-speech sounds, and the right posterior STP for non-speech sounds.

Subsequent statistical analysis of ROIs over the bilateral middle portion of the STP separate for N1a and N1b time windows revealed that current density values were strongly lateralized. A $3 \times 2 \times 2$ ANOVA with the factors temporal modulation $(5,30,60 \mathrm{~ms})$, hemisphere (left/right) 


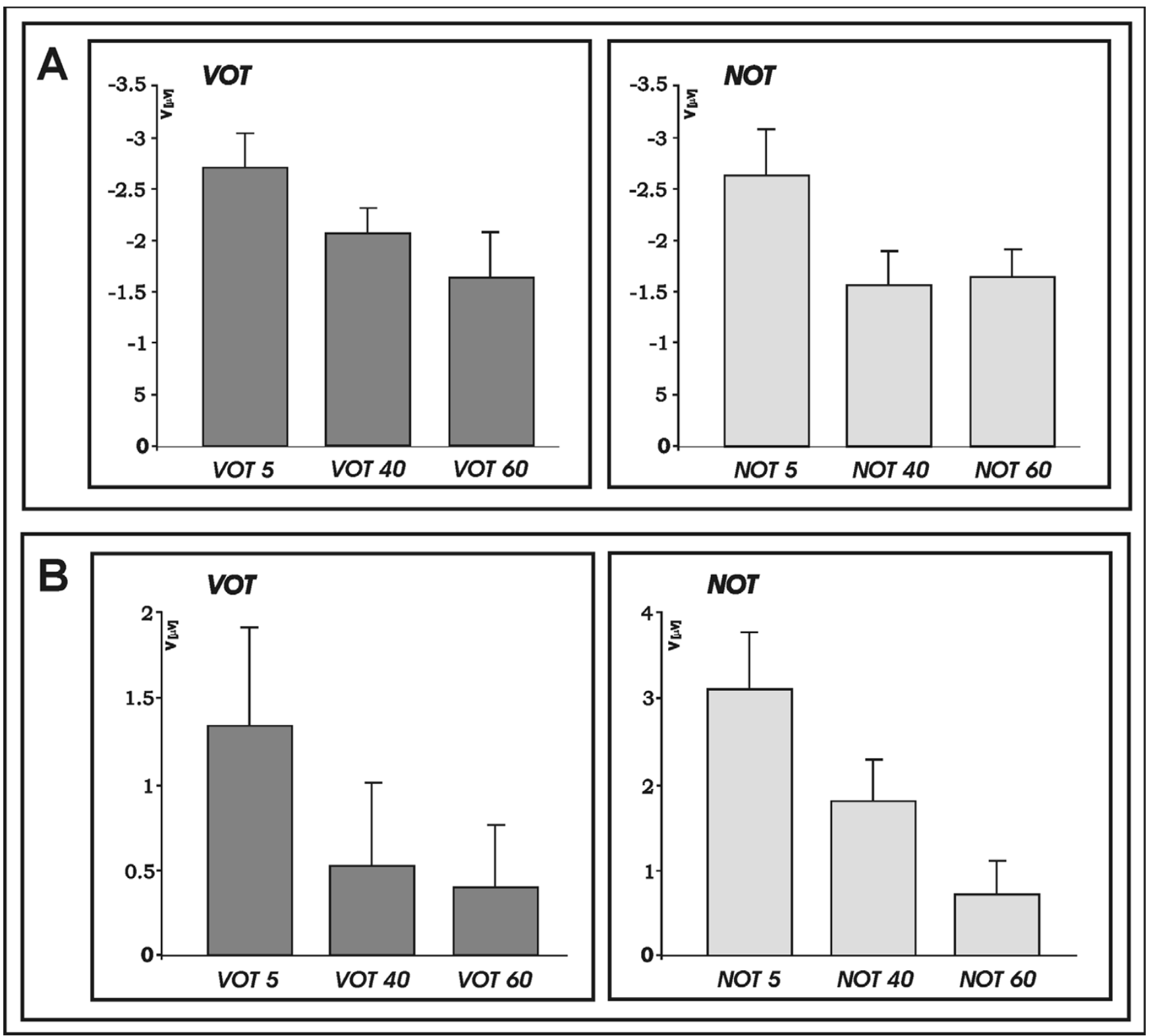

Figure 4

a: Plots of mean amplitude for NIa separate for VOT and NOT stimuli. b: Plots of mean amplitude for NIb separate for VOT and NOT stimuli.

and speechness (VOT/NOT) revealed a significant main effect of the factor hemisphere $(\mathrm{F}(1,17)=18.64$, $\mathrm{p}<0.001)$ for the N1a as well as for the N1b time window $(F(1,17)$ $=27.97, \mathrm{p}<0.001)$ demonstrating stronger responses over the left as compared to the right primary auditory cortex. Figure 6 shows current density values during the processing of VOT and NOT stimuli collapsed over the temporal modulations and extracted from the left and right primary auditory cortex.
The analysis for the posterior portion of the STP showed no significant main effect or an interaction for the N1a time window. For the N1b time window, analysis showed a significant main effect of the factor hemisphere $(\mathrm{F}(1,17)$ $=5.55, \mathrm{p}<0.05)$ indicating stronger responses over the left as compared to the right posterior STP. Figure 7 shows current density values during the processing of VOT and NOT stimuli extracted from the left and right posterior portion of the STP. 
Table I: Results of ANOVAs with the factor NOT and VOT for TW I and TW II

\begin{tabular}{|c|c|c|c|c|c|c|}
\hline \multirow[t]{2}{*}{ Factor } & \multirow[b]{2}{*}{ df } & \multirow[b]{2}{*}{ F-value } & \multirow[b]{2}{*}{ p-value } & \multicolumn{3}{|c|}{ linear contrast } \\
\hline & & & & df & F-value & p-value \\
\hline \multicolumn{7}{|l|}{ Time window I (NI) } \\
\hline VOT & 1.61 & 5.34 & 0.01 & I & 9.39 & 0.007 \\
\hline NOT & 1.81 & 8.14 & 0.001 & I & 15.53 & 0.001 \\
\hline \multicolumn{7}{|l|}{ Time window II (N2) } \\
\hline VOT & 1.79 & 3.84 & 0.03 & I & 4.98 & 0.04 \\
\hline NOT & 1.24 & 18.09 & 0.000 & I & 24.18 & 0.000 \\
\hline
\end{tabular}

\section{Discussion}

One of the key questions in understanding the nature of speech perception is to what extent the human brain has unique speech-specific mechanisms or to what degree it processes sounds equally depending on their acoustic properties. In the present study we showed that the characteristic AEP waveform in response to consonant-vowelsyllables shows an almost identical spatio-temporal pattern as in response to non-speech sounds with similar temporal characteristics. The amplitudes of the N1a and N1b component of the auditory evoked potentials significantly correlated with the duration of the VOT in CV-syllables and analogously, with the duration of the NOT in non-speech sounds. Furthermore, current density maps of the N1a and N1b time windows indicate overlapping neural distribution of these components originating from the same sections over the superior temporal plane that accommodates auditory cortex. For the analysis of the middle portion of the STP incorporating the primary audi- tory cortex, we revealed asymmetric activations that point to a stronger involvement of left supratemporal plane regardless of TW, speechness or temporal modulation. For the posterior part of the STP, the analysis of the current density values revealed a bilateral activation pattern during the N1a time window and a leftward asymmetry during the N1b time window for both the perception of speech and non-speech sounds.

In general, our data are in line with former electrophysiological studies investigating the processing of brief auditory cues but delivers novel insight in that it demonstrates a strong preference of the left middle and posterior auditory cortex for rapidly modulating temporal information by means of a low-resolution source estimation approach. Using MEG, it has been demonstrated that the AEP response to speech sounds exhibits an N100m, which is followed by a $\mathrm{N} 200 \mathrm{~m}$ at around $200-210 \mathrm{~ms}$ [42]. It has been proposed that the $\mathrm{N} 200 \mathrm{~m}$ is specific to acoustic

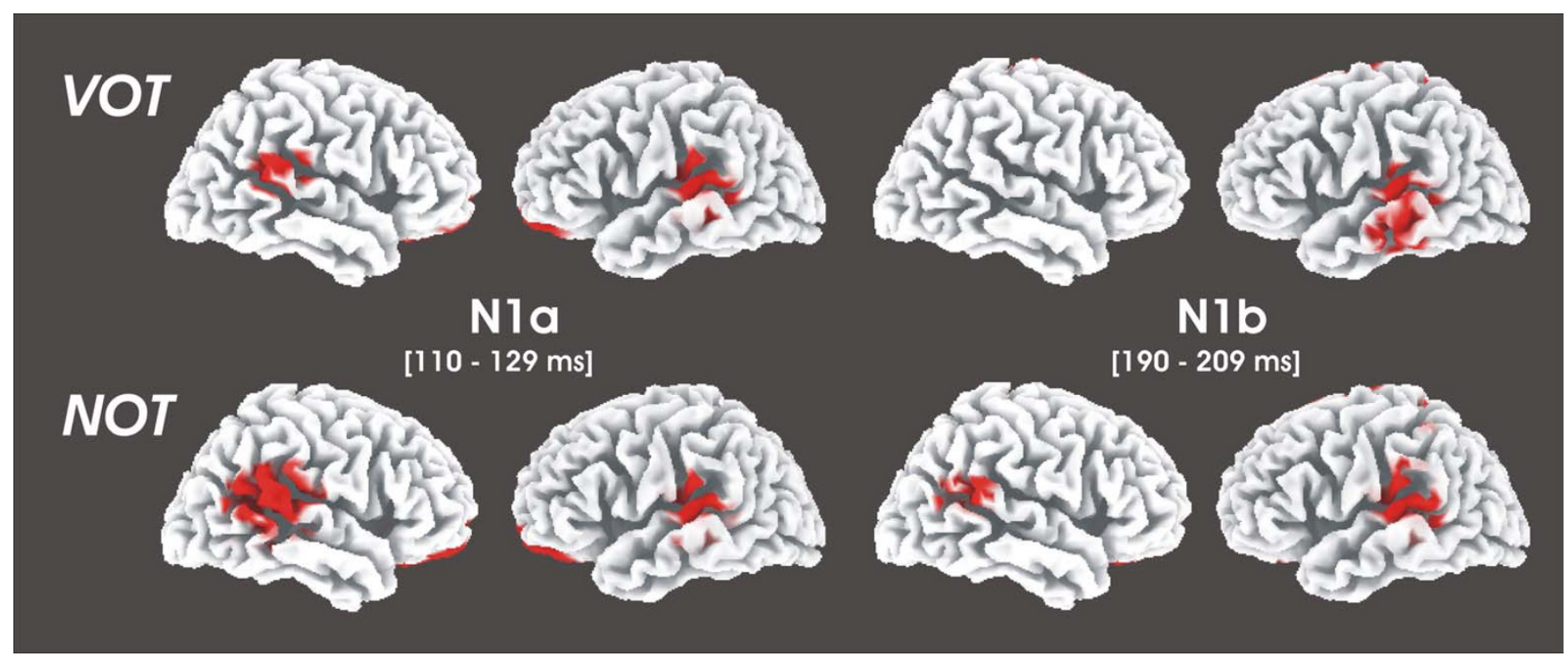

\section{Figure 5}

Grand average $(n=18)$ three dimensional LORETTA - based current density maxima for AEP components NI and N2. (Threshold: 0.001 prop. $\mu \mathrm{A} / \mathrm{mm}^{2}$ ). 
Table 2: Current density maxima $\left[\mu \mathrm{A} / \mathrm{mm}^{2}\right]^{*} 10^{-3}$ in response to speech (VOT) and non-speech (NOT) sounds

\begin{tabular}{|c|c|c|c|c|c|c|c|}
\hline Component & Condition & Brain Region & Current density value & Hemisphere & $x$ & $Y$ & Z \\
\hline \multirow[t]{6}{*}{$\mathrm{NIa}$} & VOT & Cingulum & 1.74 & & -3 & 45 & I \\
\hline & & STG & 1.39 & L & -59 & -32 & 8 \\
\hline & & & 1.30 & $R$ & 60 & -39 & 15 \\
\hline & NOT & Cingulum & 2.70 & & -3 & 45 & I \\
\hline & & STG & 1.50 & L & -59 & -32 & 8 \\
\hline & & & 1.78 & $R$ & 60 & -39 & 15 \\
\hline \multirow[t]{5}{*}{ NIb } & VOT & Cingulum & 1.74 & & -3 & 45 & I \\
\hline & & STG & 1.39 & L & -59 & -32 & 8 \\
\hline & NOT & Cingulum & 2.70 & & -3 & 52 & I \\
\hline & & STG & 1.50 & L & -59 & -32 & 8 \\
\hline & & & 1.78 & $\mathrm{R}$ & 60 & -39 & 15 \\
\hline
\end{tabular}

parameters available in vowels, since acoustic, rather than phonetic, features of the stimulus triggered the N200m. Sharma and colleagues showed that the typical change in the AEP waveform morphology from single to double peaked N1 components is not a reliable indicator of perception of voicing contrasts in syllable-initial position [3]. In other words, a double-peak onset response cannot be considered a cortical correlate of the perception of voicelessness. Rather, it depends on the acoustic properties of the sound signal. For the perception of consonants with the same place of articulation, the critical acoustic feature that distinguishes between these consonants is the time between the burst at consonant initiation and the onset of voicing (VOT). Similarly, in the case of non-speech sounds the critical acoustic feature is the time (silent gap) between the trailing and leading noise elements. In both cases the ability to perform the task requires the listener to perceptually segregate the two sounds (or their onsets) in time, which in turn requires that the brain have temporally segregated responses to the two events (or their onsets) [43]. As demonstrated by the present data, overlapping cortical excitement was found for the detection of temporal cues in both speech and non-speech sounds. Therefore, our data support the notion of similar mechanisms underling the perception of auditory events that are
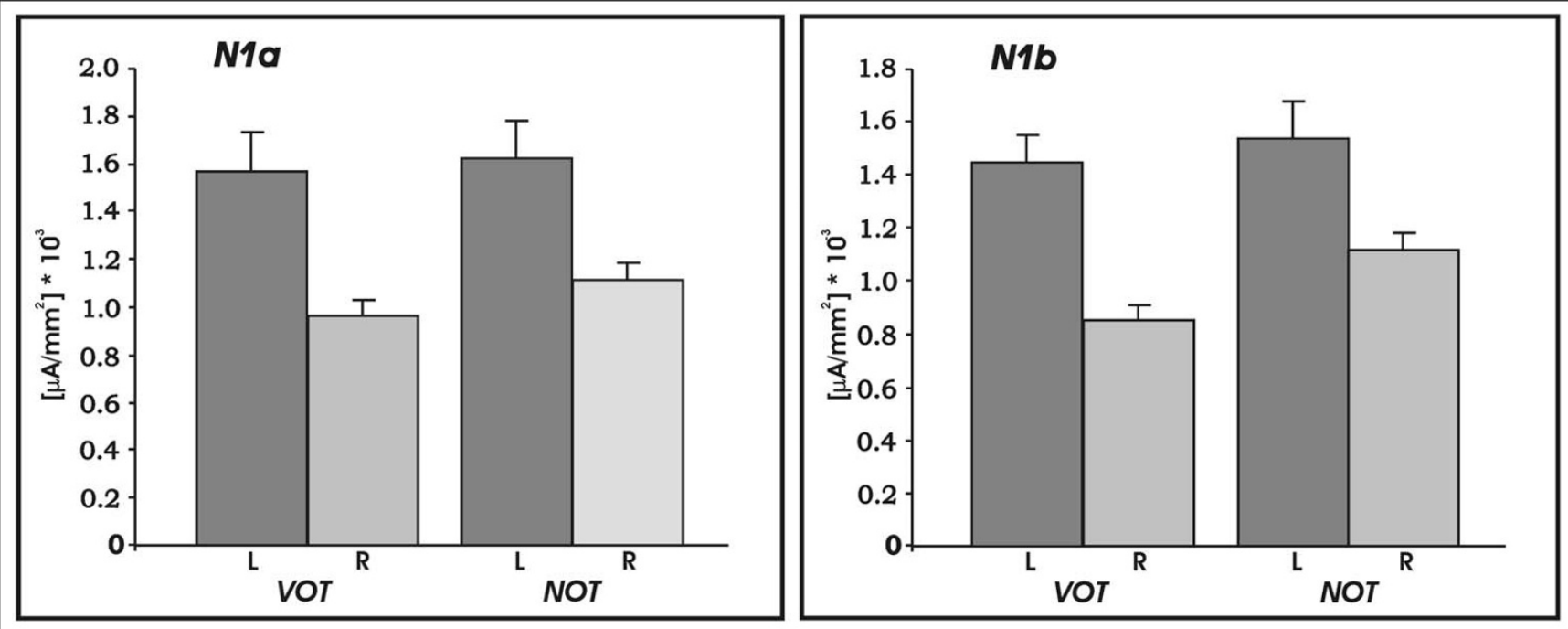

Figure 6

Plots of mean current density values obtained by the anatomically defined ROI analysis, separate for the left and right middle portion of the supratemporal plane (BA4I): Left panel shows date for NIa (TW I) and the right panel shows data for NIb (TW II). 


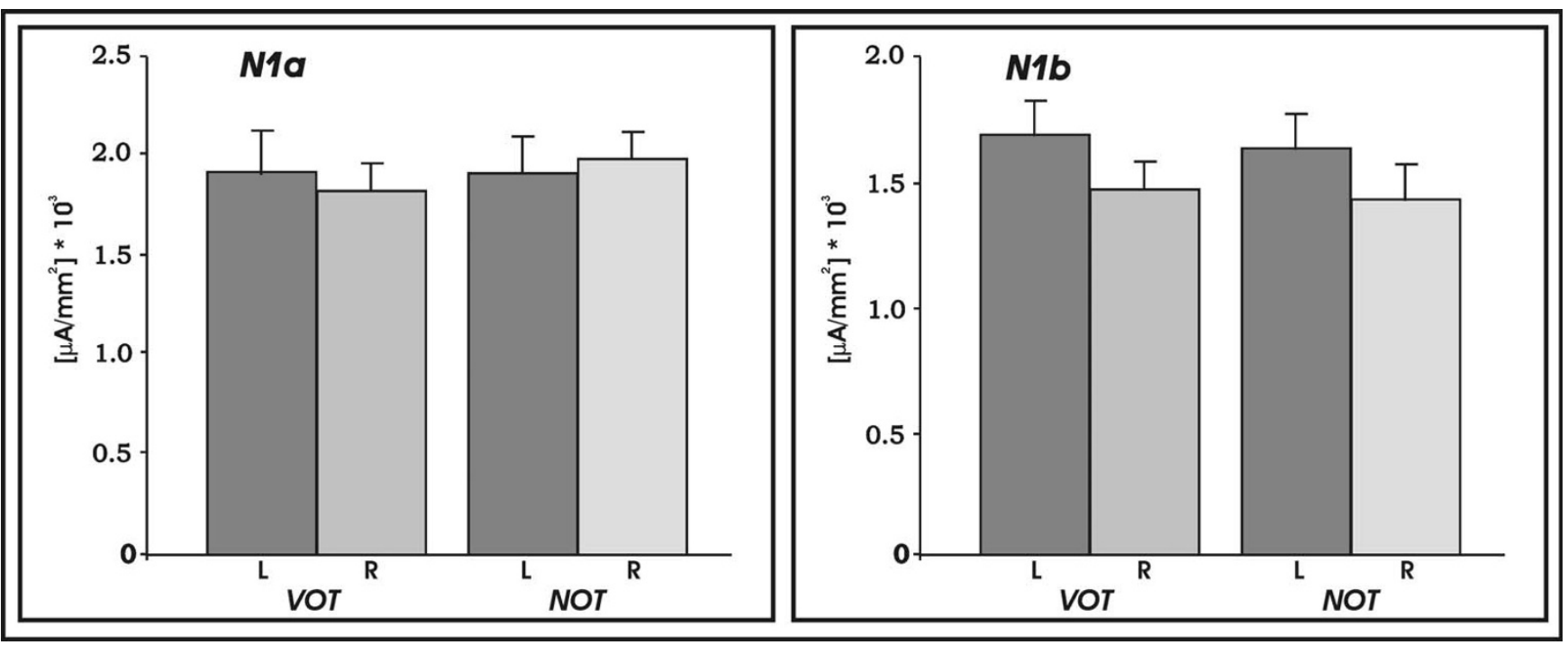

Figure 7

Plots of mean current density values obtained by the anatomically defined ROI analysis, separate for the left and right posterior portion of the supratemporal plane (post BA42): Left panel shows date for NIa (TW I) and the right panel shows data for NIb (TW II).

equal in temporal acoustic structure but differ in their linguistic meaning.

It has been suggested that the primary auditory cortex is specifically involved in the perceptual elaboration of sounds with durations or spacing within a specific temporal grain [43] and this suggestion has been confirmed by studies demonstrating that primary auditory cortex evoked responses reflect encoding of VOT $[9,11,23,24]$. Furthermore, Heschl's gyrus (HG) is known to display a leftward structural asymmetry [44-47]. This asymmetry is related to a larger white matter volume of the left as compared to the right HG $[44,48]$, as well as to asymmetries at the cellular level [49-52]. It has been hypothesized that this leftward asymmetry of the HG is related to a more efficient processing of rapidly changing acoustic information, which is relevant in speech perception [53].

The posterior part of the left STP that partly covers the planum temporale (PT) has also been associated with competence to mediate spectro-temporal integration during auditory perception $[54,55]$. In particular, the left posterior auditory cortex plays a prominent role when speech relevant auditory information has to be processed $[26,27,56]$. Akin to the primary auditory cortex that resides in $\mathrm{HG}$, the posterior STP also has structural leftward asymmetry $[57,58]$, which indicates a relationship between this brain region and the leftward lateralized specific functions relevant to speech perception.
The present study revealed a clear asymmetrical response pattern over the posterior supratemporal plane during the N1b (TW II) for both the NOT and the VOT condition. Interestingly, we also observed a symmetrical response pattern during the N1a component (TW I) over the same cortical portion. In this vein are the findings of Rimol and colleagues who reported that the well established right-ear advantage (REA, indicative of a left hemisphere superiority) during a dichotic listening (DL) syllable task is found to be significantly affected by VOT [59]. More elaborately, the authors compellingly demonstrate that the REA reverses into a left-ear advantage under certain constellations of different VOT in the DL tasks. In addition, a recent study applying LORETA source estimation revealed differentially lateralized responses over the posterior STP contingent upon constellations of different VOT using the same DL task [24]. Thus, it can be concluded that the degree of asymmetry during DL is influenced by the length of the VOT as evidenced by both behavioural and electrophysiological measures. Based on these findings it could be assumed that the early symmetric effect over the posterior STP might be related to the differentially asymmetric effects of VOT length since our source estimation approach did not specifically emphasize this effect.

As mentioned above, a long lasting question in auditory speech research concerns the nature of the VOT cue and asks to what extent the VOT is processed by specialized speech mechanisms or by more basic acoustically tuned mechanisms [60]. Evidence for a specialized speech processing stems from the well known observation that 
the perception of series of (synthetic) speech stimuli varying continuously in VOT is almost categorical [61]. This effect of categorical perception implicates that for a series of stimuli the percept exists only in one of two categories: the voiced and voiceless stop. Furthermore, listeners can discriminate differences in VOT considerably better when two stimuli lie in different phonetic categories than when the two stimuli are from the same category. However, the effect of categorical perception also exists for non-speech stimuli [60]. As suggested by Phillips (1993), as far as the stimulus representation in the primary auditory cortex is concerned, speech may be "special" only in the sense that spoken language is the most obvious stimulus in which the identification of the elements is dependent on temporal resolution [43]. In fact, data of the present study evidence that the middle and posterior auditory cortex especially of the left hemisphere is significantly involved in the processing of the acoustical features critical for the processing of temporal cues in both speech and nonspeech sounds.

This conclusion corroborates recent fMRI research, but in addition demonstrates that EEG in combination with low-resolution tomography could be considered an ideal alternative to map the spatio-temporal patterns of speech perception. In a way, this approach outperforms the fMRI technology because it evidently demonstrates the temporal subtlety of elemental acoustic processing reflected by differential sensitivity and neural distribution of succeeding N1a and N1b responses to brief speech and speechlike stimuli. Of course, one should bear in mind that spatial resolution of electrophysiologically based localization methods is inferior to modern brain imaging techniques. Thus, one should by no means feel tempted to interpret the activation maps provided by LORETA in an fMRI-like manner. However, it has been proven that low-resolution tomography is capable of reliably distinguishing between sources originating from distinct sections of the superior temporal region [62]. This holds particularly true if lowresolution tomography is used to examine electrophysiological responses emerging from the left or right hemispheres [63].

\section{Conclusion}

In essence, the present study delivers further evidence for the prominent role of the middle and posterior left supratemporal plane in the perception of rapidly changing cues, which is thought to be an essential device underlying speech perception $[53,64,65]$.

\section{Authors' contributions}

TZ designed the experimental paradigm, performed the data acquisition and statistical analysis and drafted the manuscript
LJ contributed to the hypothesis, design, results, discussion, and to the preparation of the manuscript

MM conceived of the study, participated in its design and coordination and contributed to the manuscript

All authors read and approved the final manuscript.

\section{Acknowledgements}

This work was supported by Swiss National Science Foundation Grant No. 46234103 (TZ) and Swiss SNF 46234I0I (MM).

\section{References}

I. Davis $\mathrm{MH}$, Johnsrude IS: Hearing speech sounds: Top-down influences on the interface between audition and speech perception. Hear Res 2007, 229:|32-147.

2. Lisker L, Abramson AS: Across language study of voicing in initial stops: Acoustical measurements. Word 1964, 20:384-4I I.

3. Sharma A, Marsh CM, Dorman MF: Relationship between NI evoked potential morphology and the perception of voicing. J Acoust Soc Am 2000, 108:3030-3035.

4. Sharma A, Dorman MF: Cortical auditory evoked potential correlates of categorical perception of voice-onset time. J Acoust Soc Am 1999, 106:1078-1083.

5. Steinschneider M, Volkov IO, Noh MD, Garell PC, Howard MA III: Temporal encoding of the voice onset time phonetic parameter by field potentials recorded directly from human auditory cortex. J Neurophysiol 1999, 82:2346-2357.

6. Roman S, Canevet G, Lorenzi C, Triglia JM, Liegeois-Chauvel C: Voice onset time encoding in patients with left and right cochlear implants. Neuroreport 2004, 15:601-605.

7. Giraud K, Demonet JF, Habib M, Marquis P, Chauvel P, LiegeoisChauvel C: Auditory evoked potential patterns to voiced and voiceless speech sounds in adult developmental dyslexics with persistent deficits. Cereb Cortex 2005, I 5: I 524-I534.

8. Steinschneider M, Reser D, Schroeder CE, Arezzo JC: Tonotopic organization of responses reflecting stop consonant place of articulation in primary auditory cortex (AI) of the monkey. Brain Res 1995, 674:147-152.

9. Steinschneider M, Volkov IO, Fishman YI, Oya H, Arezzo JC, Howard MA III: Intracortical responses in human and monkey primary auditory cortex support a temporal processing mechanism for encoding of the voice onset time phonetic parameter. Cereb Cortex 2005, I 5:170-186.

10. McGee T, Kraus N, King C, Nicol T, Carrell TD: Acoustic elements of speechlike stimuli are reflected in surface recorded responses over the guinea pig temporal lobe. J Acoust Soc Am 1996, 99:3606-36|4.

II. Liegeois-Chauvel C, de Graaf JB, Laguitton V, Chauvel P: Specialization of left auditory cortex for speech perception in man depends on temporal coding. Cereb Cortex 1999, 9:484-496.

12. Efron R: Temporal Perception, Aphasia and D'ej'a vu. Brain 1963, 86:403-424.

13. Swisher L, Hirsh IJ: Brain damage and the ordering of two temporally successive stimuli. Neuropsychologia 1972, I0:137-152.

14. Tallal P, Piercy M: Defects of non-verbal auditory perception in children with developmental aphasia. Nature 1973, 24l:468-469.

15. Tallal P, Stark RE: Speech acoustic-cue discrimination abilities of normally developing and language-impaired children. J Acoust Soc Am 1981, 69:568-574.

16. Tallal P: Auditory temporal perception, phonics, and reading disabilities in children. Brain Lang 1980, 9:182-198.

17. Tallal P, Stark RE, Kallman C, Mellits D: Developmental dysphasia: relation between acoustic processing deficits and verbal processing. Neuropsychologia 1980, 18:273-284.

18. Tallal P, Miller S, Fitch RH: Neurobiological basis of speech: a case for the preeminence of temporal processing. Ann $N Y$ Acad Sci 1993, 682:27-47.

19. Breier JI, Gray L, Fletcher JM, Diehl RL, Klaas P, Foorman BR, Molis $M R$ : Perception of voice and tone onset time continua in chil- 
dren with dyslexia with and without attention deficit/hyperactivity disorder. J Exp Child Psychol 200I, 80:245-270.

20. Von Steinbuchel $N$ : Temporal ranges of central nervous processing: clinical evidence. Exp Brain Res 1998, I 23:220-233.

21. Tallal P, Miller SL, Bedi G, Byma G, Wang X, Nagarajan SS, Schreiner C, Jenkins WM, Merzenich MM: Language comprehension in language-learning impaired children improved with acoustically modified speech. Science 1996, 27 I:81-84.

22. Merzenich MM, Jenkins WM, Johnston P, Schreiner C, Miller SL, Talla $P$ : Temporal processing deficits of language-learning impaired children ameliorated by training. Science 1996, 27I:77-8I.

23. Trebuchon-Da FA, Giraud K, Badier JM, Chauvel P, Liegeois-Chauvel $C$ : Hemispheric lateralization of voice onset time (VOT) comparison between depth and scalp EEG recordings. Neuroimage $2005,27: 1-14$.

24. Sandmann P, Eichele T, Specht K, Jancke L, Rimol LM, Nordby H, Hugdahl K: Hemispheric asymmetries in the processing of temporal acoustic cues in consonant-vowel syllables. Restor Neurol Neurosci 2007, 25:227-240.

25. Papanicolaou AC, Castillo E, Breier JI, Davis RN, Simos PG, Diehl RL: Differential brain activation patterns during perception of voice and tone onset time series: a MEG study. Neuroimage 2003, 18:448-459.

26. Zaehle T, Wustenberg T, Meyer M, Jancke L: Evidence for rapid auditory perception as the foundation of speech processing: a sparse temporal sampling fMRI study. Eur J Neurosci 2004, 20:2447-2456

27. Meyer M, Zaehle T, Gountouna VE, Barron A, Jancke L, Turk A Spectro-temporal processing during speech perception involves left posterior auditory cortex. Neuroreport 2005, 16:1985-1989.

28. Jancke L, Wustenberg $\mathrm{T}$, Scheich $\mathrm{H}$, Heinze HJ: Phonetic perception and the temporal cortex. Neuroimage 2002, I5:733-746.

29. Zaehle T, Geiser E, Alter K, Jancke L, Meyer M: Segmental processing in the human auditory dorsal stream. Brain Res 2007 in press.

30. Pascual-Marqui RD, Lehmann D, Koenig T, Kochi K, Merlo MC, Hell $D$, Koukkou M: Low resolution brain electromagnetic tomography (LORETA) functional imaging in acute, neurolepticnaive, first-episode, productive schizophrenia. Psychiatry Res 1999, 90:169-179.

31. Naatanen R, Picton T: The NI wave of the human electric and magnetic response to sound: a review and an analysis of the component structure. Psychophysiology 1987, 24:375-425

32. Picton TW, Skinner CR, Champagne SC, Kellett AJ, Maiste AC Potentials evoked by the sinusoidal modulation of the amplitude or frequency of a tone. J Acoust Soc Am 1987, 82: 165- I 78.

33. Ostroff JM, Martin BA, Boothroyd A: Cortical evoked response to acoustic change within a syllable. Ear Hear 1998, 19:290-297.

34. Sharma A, Dorman MF: Neurophysiologic correlates of crosslanguage phonetic perception. J Acoust Soc Am 2000, 107:2697-2703.

35. Pratt H, Starr A, Michalewski HJ, Bleich N, Mittelman N: The N I complex to gaps in noise: effects of preceding noise duration and intensity. Clin Neurophysiol 2007, I I 8: 1078-1087.

36. SoundForge 4.51999 [http://www.sonicfoundry.com]. Sonic Foundry Inc.

37. PRAAT 4.6 2007 [http://www.fon.hum.uva.nl/praat/]

38. Pascual-Marqui RD, Michel CM, Lehmann D: Low resolution electromagnetic tomography: a new method for localizing electrical activity in the brain. Int I Psychophysiol 1994, I 8:49-65.

39. Pascual-Marqui RD, Esslen M, Kochi K, Lehmann D: Functional imaging with low-resolution brain electromagnetic tomography (LORETA): a review. Methods Find Exp Clin Pharmacol 2002, 24 Suppl C:9l-95.

40. Talairach J, Tournoux P: Co-palanar Stereotaxis Atlas of the Human Brain New York, Thieme; 1988.

4I. ROI extractor tool box 2005 [http://www.novatecheeg.com/ Downloads.html].

42. Kaukoranta E, Hari R, Lounasmaa OV: Responses of the human auditory cortex to vowel onset after fricative consonants. Exp Brain Res 1987, 69:19-23.

43. Phillips DP: Neural representation of stimulus times in the primary auditory cortex. Ann N Y Acad Sci 1993, 682: I04-I I8.
44. Penhune VB, Zatorre RJ, MacDonald JD, Evans AC: Interhemispheric anatomical differences in human primary auditory cortex: probabilistic mapping and volume measurement from magnetic resonance scans. Cereb Cortex 1996, 6:661-672.

45. Penhune VB, Cismaru R, Dorsaint-Pierre R, Petitto LA, Zatorre RJ: The morphometry of auditory cortex in the congenitally deaf measured using MRI. Neuroimage 2003, 20: I2 I5- I 225.

46. Rademacher J, Caviness VS Jr., Steinmetz H, Galaburda AM: Topographical variation of the human primary cortices: implications for neuroimaging, brain mapping, and neurobiology. Cereb Cortex 1993, 3:313-329.

47. Dorsaint-Pierre R, Penhune VB, Watkins KE, Neelin P, Lerch JP, Bouffard $M$, Zatorre RJ: Asymmetries of the planum temporale and Heschl's gyrus: relationship to language lateralization. Brain 2006, 129:1164-1176.

48. Sigalovsky IS, Fischl B, Melcher JR: Mapping an intrinsic MR property of gray matter in auditory cortex of living humans: a possible marker for primary cortex and hemispheric differences. Neuroimage 2006, 32:1524-1537.

49. Hutsler JJ, Gazzaniga MS: Acetylcholinesterase staining in human auditory and language cortices: regional variation of structural features. Cereb Cortex 1996, 6:260-270.

50. Seldon HL: Structure of human auditory cortex. III. Statistical analysis of dendritic trees. Brain Res 1982, 249:21 I-221.

5I. Seldon HL: Structure of human auditory cortex. II. Axon distributions and morphological correlates of speech perception. Brain Res 1981, 229:295-310.

52. Seldon HL: Structure of human auditory cortex. I. Cytoarchitectonics and dendritic distributions. Brain Res 1981, 229:277-294.

53. Zatorre RJ, Belin P: Spectral and temporal processing in human auditory cortex. Cereb Cortex 200I, I I:946-953.

54. Griffiths TD, Warren JD: The planum temporale as a computational hub. Trends Neurosci 2002, 25:348-353.

55. Warren JD, Jennings AR, Griffiths TD: Analysis of the spectral envelope of sounds by the human brain. Neuroimage 2005, 24:1052-1057.

56. Geiser E, Zaehle T, Jancke L, Meyer M: The Neural Correlate of Speech Rhythm as Evidenced by Metrical Speech Processing: A Functional Magnetic Resonance Imaging Study. J Cogn Neurosci 2007

57. Anderson B, Southern BD, Powers RE: Anatomic asymmetries of the posterior superior temporal lobes: a postmortem study. Neuropsychiatry Neuropsychol Behav Neurol 1999, I 2:247-254.

58. Galuske RA, Schlote W, Bratzke H, Singer W: Interhemispheric asymmetries of the modular structure in human temporal cortex. Science 2000, 289:1946-1949.

59. Rimol LM, Eichele T, Hugdahl K: The effect of voice-onset-time on dichotic listening with consonant-vowel syllables. Neuropsychologia 2006, 44:191-196.

60. Pisoni DB: Identification and discrimination of the relative onset time of two component tones: implications for voicing perception in stops. J Acoust Soc Am 1977, 61: I352-I36I.

61. Abramson AS, Lisker L: Discriminability along the voicing continuum: cross-language tests. In 6th International Congress of Phonetics Sciences Prague, Academia; 1970:569-573.

62. Meyer M, Baumann S, Jancke L: Electrical brain imaging reveals spatio-temporal dynamics of timbre perception in humans. Neuroimage 2006, 32:1510-1523.

63. Sinai $A$, Pratt $H$ : High-resolution time course of hemispheric dominance revealed by low-resolution electromagnetic tomography. Clin Neurophysiol 2003, I I 4: I I8I-I I 88.

64. Poeppel $D$ : The analysis of speech in different temporal integration windows: cerebral lateralization as 'asymmetric sampling in time'. Speech Commun 2003, 41:245-255.

65. Hickok G, Poeppel D: The cortical organization of speech processing. Nat Rev Neurosci 2007, 8:393-402. 